\title{
Analysis of Mistyping in Hardware Keyboard
}

\author{
Ryuki Komatsu ${ }^{\mathrm{a}, *}$, Yoshihisa Nakatoh $^{\mathrm{a}}$ \\ ${ }^{a}$ Kyushu Institute of Technology \\ 1-1, Sensui-cho, Tobata-ku, Kitakyushu-shi, Fukuoka, 804-0015, Japan \\ *nakatoh@ecs.kyutech.ac.jp
}

\begin{abstract}
In recent years, aging has become a social problem in Japan, and employment of elderly people is also increasing accordingly. About $80 \%$ of the elderly have a job with many opportunities to touch the personal computer. However, it is difficult to work for the elderly because accuracy of the operation of the personal computer is required. Therefore, we pay attention to the keyboard input which is the basis of personal computer operation, and are studying the correction method of mistyping which occurs at the time of input. As a preliminary study for correcting mistypes this time, we analyzed for 20 s accustomed to personal computer operation. The results showed common features among all the subjects, and characteristic mistypes were also seen in each. From this result, it was found that it is necessary to grasp personal characteristics for correction.
\end{abstract}

Keywords: Keyboard, Personal computer, Typing mistake, User interface, Ergonomics.

\section{Introduction}

In recent years, the aging has become a social problem in Japan, and the employment of elderly people is also increasing accordingly. $80 \%$ of the occupations in which elderly people are employed are technical and professional, management or office work, and there are many opportunities to operate personal computers ${ }^{(1)}$. Moreover, as of the year of 2008, the personal computer ownership rate of household head age 65 years old or more is much higher than $50 \%{ }^{(2)}$. However, for elderly people, personal computers are difficult to operate and need to be easy to use.

Therefore, in this study, as a preliminary study for correcting mistyping, analyze what feature of mistyping are present in young people who are familiar with personal computers, and whether there is individual difference or not.

\section{Classification of Mistype}

As an analysis method of mistyping, divide the pattern of input errors into six ${ }^{(3)}$. Table 1 is an example of each input error. A detailed explanation of each mistake is described below.

(a) Replacement

The first one is "replacement". Replacement is to press another key when you must press correct key. Looking at an example, you can see that " $U$ " is input where "I" must be input.

(b) Removal

The second is "removal". Removal means that characters what must be input are not input. Looking at an example, you can see that there is one $\mathrm{N}$ short.

(c) Exchange

The third one is "exchange". exchange is to press the next key earlier than the key what must be typed first when entering a character string. Looking at an example, you can see that the locations of $\mathrm{C}$ and $\mathrm{H}$ are interchanged.

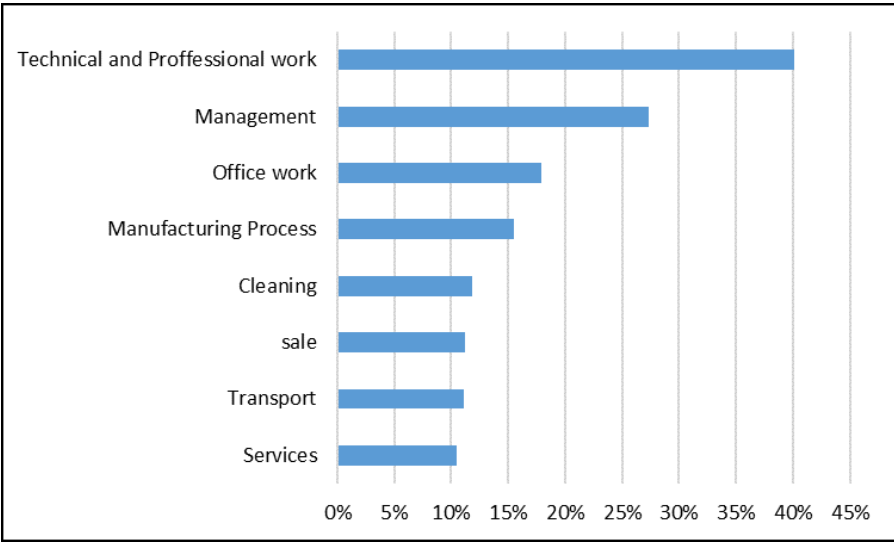

Fig. 1. Work of elderly people 
(d) Insertion

The fourth one is "Insertion". Insertion is that inappropriate characters get into the correct character string. In addition, there are two types of insertion, "involvement" and "continuation".

(e) Involvement

The fifth one is "involvement". Involvement is to press also the adjacent key when pressing the appropriate key. The involvement belongs to the type of "Insertion". Looking at an example, you can see that $\mathrm{O}$ next to $\mathrm{I}$ is unnecessarily entered.

\section{(f) Repetition}

The sixth is "repetition". Repetition is to push the same key unnecessarily twice or more in succession. Repetition belongs to the type of "Insertion". Looking at an example, you can see that $\mathrm{N}$ has been entered more than once.

Generally, typing errors can be classified by the above six types, but if there are mistakes not belonging to six types, it belong to the type that seems to be the closest.

\section{Evaluation of Experimentation}

Using the above classification method, we conducted an experiment to analyze what feature of mistyping exist for each subjects. Experimental conditions and experimental results are as follows.

\subsection{Experimental Condition}

Ask twelve general men in their 20 s to input 100 words per experiment, and have them do 10 tries (1000 words in total). In order to have natural input, it is permitted that using the delete operation (BackSpace) of characters and checking the keyboard. Also, when experimenting, we measure the time to induce mistakes and made them conscious to have them input as quickly as possible. In this time, input style is hiragana. This is to eliminate the conversion error. About 3000 words such as verbs, adjectives, and nouns are prepared for the experiment, and 100 words are input at random each time. The experimental procedure is shown in Fig. 2.

\subsection{Experimental Result}

Fig. 3 shows the experimental results for all subjects and Fig. 4 shows the experimental results for each subjects "Replacement" was common input error for all subjects. In particular, replacement by "O" and "I","I" and "U" occurred frequently because it is used frequently in Japanese input
Table 1. Example of mistyping

\begin{tabular}{|c|c|}
\hline Type of Miss & e.g.:KONNNICHIHA(こんにちは) \\
\hline Replacement & KONNNUCHIHA(こんぬちは) \\
\hline Removal & KONNICHIHA(こんいちは) \\
\hline Exchange & 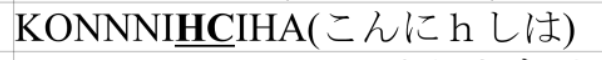 \\
\hline \multicolumn{2}{|c|}{$\begin{array}{c}\text { InvolvementKONNNIOCHIHA(こんにおちは) } \\
\text { Repetition KONNNNICHIHA(こんんいちは) }\end{array}$} \\
\hline
\end{tabular}


Fig. 2. Experiment procedure

and the key arrangement is next to each other. Also, there were characteristic mistypes for each subject. Characteristic erroneous inputs of each subject are shown in Table 2. It is assumed that these feature are greatly related to typing accuracy and skill of the subjects. The analysis results of each subject are described below.

(a) $\mathrm{A}$

Subject A had the average trial time of 5 minutes 37 seconds and the total number of mistypes was 398, the second most among all subjects. It seems to be because it prompts for input by measuring time. In addition, since Subject A is performing blind touch roughly and its typing is not performed accurately, it is considered that the number 
of mistakes has increased. As a characteristic mistake of subject A, replacement errors of "K" and "S", "N" and "I" were seen. These are thought to be caused by a certain character string.

(b) $\mathrm{B}$

Subject B had the average trial time of 7 minutes 4 seconds and the total number of mistypes was as small as 161, indicating that accurate input was done. As a characteristic of subject $\mathrm{B}$, it was found that the rate of insertion error is considerably larger than that of other subjects. Especially, there are a lot of "involvement" in mistakes in "insertion" and these are frequently occured with "Y", "U", "I", "O", "P" of the upper right hand side. Because this is high frequency of use, and the subject himself does not accurately grasp the key placement, the possibility of inducing mistakes is considered to be increased.

(c) $\mathrm{C}$

Subject $C$ had the average trial time of 6 minutes 47 seconds and the total number of mistypes was 197 times. The subject is not seen personal features. However, it was found that the percentage of replacement errors between adjacent keys accounted for approximately $70 \%$ of total replacement mistakes, which was higher than other subjects. This is caused by subject's key hitting method. The his key input operation is performed with only the left and right middle fingers. For this reason, it seems that replacement mistakes of adjacent keys occur frequently.

(d) $\mathrm{D}$

Subject D had the average trial time of 6 minutes 2 seconds and the total number of total mistypes was 177 times. As characteristics of the subject, the percentage of replacement miss is the second most among all subjects and the rate of insertion miss is the lowest. Moreover, it was found that the removal miss rate was high. These are due to the weakness of pressing keys and concentrating their consciousness on typing flow without pressing them certainly. Furthermore, this subject has a high rate of erroneously entering "S", which is a feature not found in other subjects. This is considered to be a habit of unconsciously pushing the key of "S" which is frequently used in pressing another key.

(e) E

Subject E had the average trial time of 7 minutes 17 seconds which was slightly slower than those of the other subjects and the total number of mistypes was 212 times. The characteristic of this subject is that there are many mistakes of replacing "U" and "O". It is considered that it is easy to induce mistakes because sounds of "_ゆ(_YU)" and "_よ(_YO)" are similar and he unconsciously presses "U" which is close to "Y" when he input contracted sound such as "りょ(RYO)","きや(KYA)","みゆ(MYU)".

(f) $\mathrm{F}$

Subject $\mathrm{F}$ has the average trial time of 7 minutes 37 seconds and the total number of mistypes is as small as 128 , so it is clear that typing is performed slowly and reliably. The characteristic of this subject is that the rate of repetition miss is high. Especially, it turned out that "N" was repeated many times. This is thought to be due to erroneously pushing "N" three times at the time of input of " $(\mathrm{NN})$ ".

(g) G

Subject $\mathrm{G}$ had the average trial time of 4 minutes 13 seconds, the fastest among all subjects, and the total mistyping number was 242 times. This subject had few mistakes in replacement of "U", "I", and "O" compared to other subjects. But there were many mistakes in replacement miss of "R" and "T". This is probably because

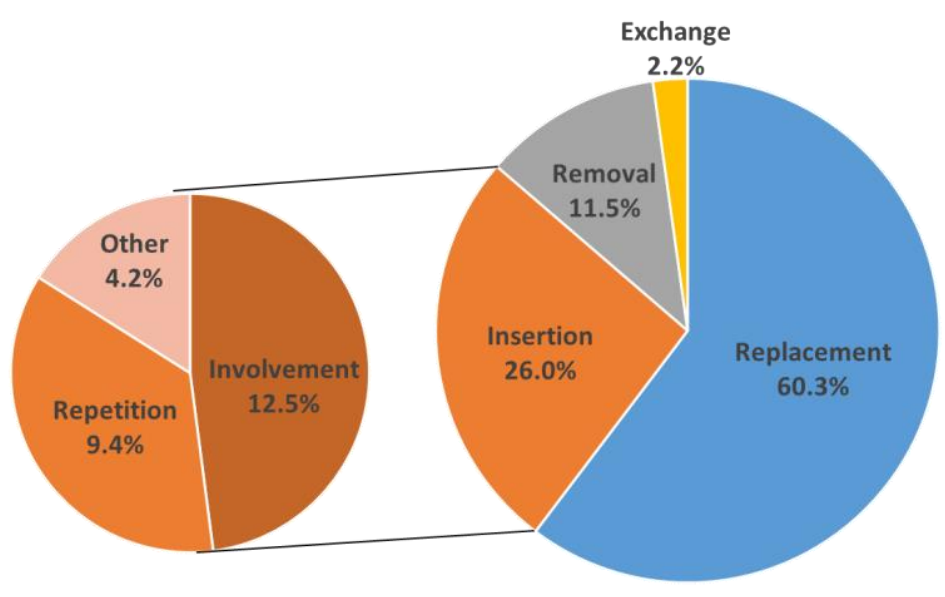

Fig. 3. Analysis results for all subjects

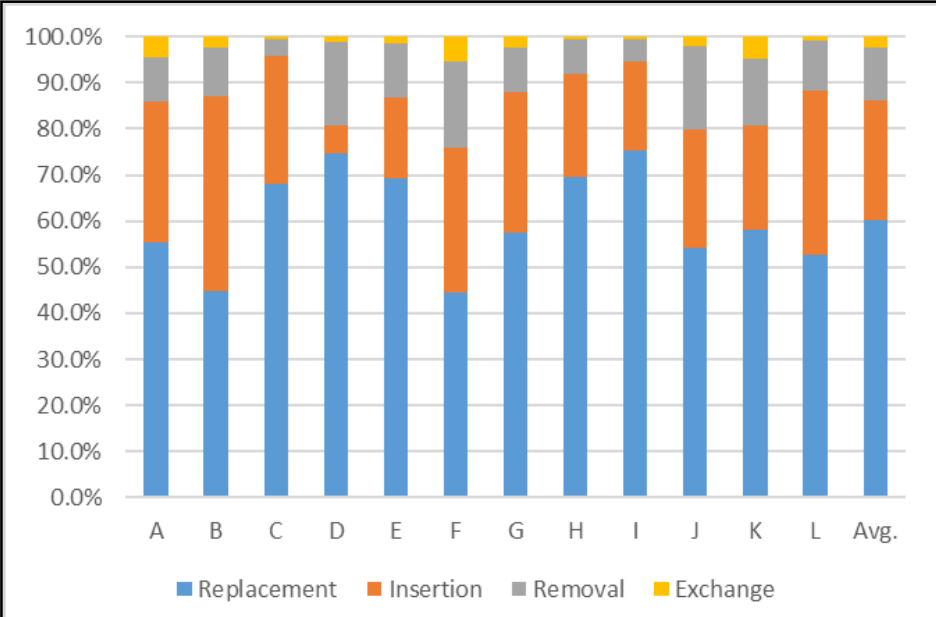

Fig. 4. Analysis results for each subjects 
the subject himself does not accurately grasp the position of "R" and "T". In addition, there was also a lot of insertion miss, and rate of involvement miss was $20 \%$ of the all miss which was larger than those of the other subjects. He concentrated on the flow as same as the subject D, grasp how to type, and input it all at once with blind touch, so that he also input the next key.

\section{(h) $\mathrm{H}$}

In subject $\mathrm{H}$, the average trial time was slightly late as 6 minutes 54 seconds, and the total number of mistypes was 208. As a feature of the subject, the mistake of replacement is as high as $70 \%$, especially the replacement miss of "U", "I" and "O" accounts for $30 \%$ of the total. It is also a feature that replacement miss of "A" and "O" frequently occurred. This is caused by unconsciously pushing the vowel key near pressed consonant key. In addition, this subject once plays the sounds of displayed word in his head and performs input. Therefore, there is a tendency to frequently mistake the inputs whose pronunciations are similar, such as "SHA" and "SHO".

(i) I

Subject I had an average trial time of 6 minutes 49 seconds and the total number of mistypes was 455, which was the largest among all subjects. As a feature of this subject, the replacement miss rate is high. It is found that the replacement miss of the adjacent key is $87 \%$, which is almost replacement error with the adjacent key. Although this is caused by grasping the approximate location of the key without grasping the accurate location.

(j) $\mathrm{J}$

Subject $\mathrm{J}$ had an average trial time as fast as 4 minutes 32 seconds, and the total number of mistypes was as small as 144, indicating that accurate and fast typing is taking place. The feature of this subject is that the removal rate is high. This is thought to be due to that the pressing strength of the key is weakened by inputting fast.

(k) $\mathrm{K}$

Subject K, like subject J, had a short average trial time of 4 minutes and 48 seconds, and the total number of mistypes was as small as 188 times. The feature of this subject is that there is a high proportion of exchange miss. It is thought that this is due to that he presses a key which is easy to input before hitting a correct character.

(l) L

Subject $\mathrm{L}$ had the shortest average trial time of 4 minutes 23 seconds and the total number of mistypes was 127 times. As a characteristic of this subject, there were many mistakes in insertion, especially the percentage of
Table 2. Features of all subjects

\begin{tabular}{|c|c|}
\hline Subject & Feature \\
\hline A & There are many mistakes in replacing “ $\mathrm{K}$ ” and “S”, " $\mathrm{N}$ ” and “ $\mathrm{I}$ ” keys \\
\hline $\mathrm{B}$ & There are many mistakes of involution \\
\hline $\mathrm{C}$ & There are many replacements between the keys on the left and right \\
\hline $\mathrm{D}$ & The frequency of replacement mistakes is the highest \\
\hline $\mathrm{E}$ & Many replacement mistakes of " $\mathrm{O}$ " and " $U$ " \\
\hline $\mathrm{F}$ & There are many continuation of “N” \\
\hline G & There are many replacement errors of "R" and "T". \\
\hline $\mathrm{H}$ & The ratio of $\mathrm{U}, \mathrm{I}$ and $\mathrm{O}$ substitution error is high. \\
\hline $\mathrm{I}$ & Most of replacement errors are next to each other. \\
\hline $\mathbf{J}$ & Removal error rate is high. \\
\hline K & Compatibility error rate is high. \\
\hline $\mathrm{L}$ & Trapped mistakes are the highest. \\
\hline
\end{tabular}

involvement was the highest among all subjects. This is due to the fast key input speed, and since it is inputting in a hurry, it is thought that the involvement frequently occurs hitting the next key.

Looking at these results, the tendency of erroneous input varies depending on individuals, and it can be seen that it is necessary to grasp the characteristics of each user and to take countermeasures in order to correct them.

\section{Conclusions}

By this research, it was possible to classify various mistypes to some extent. In addition, common miss and peculiar miss of each subject were seen, and it was confirmed that there were individual differences. In the future, as a correction method, a process of recognizing miss and correcting miss is necessary. For recognition of inputting miss, it is assumed it is possible to find miss by using morphological analysis, N-gram or other methods. And it is assumed it is possible to improve typos by using machine learning, neural networks, etc. Although we conducted experiments for adults in their 20s this time, we will conduct similar experiments for the elderly and plan to establish a method for correcting mistypes.

\section{References}

(1) Labor Policy Research and Training Organization, "Survey on employment of older people"

(2) Ministry of Public Management, Home Affairs, Posts and Telecommunications Trend Survey (28th Report)

(3) Yuzo Noda, "Survey and Analysis of Erroneous Keying Characteristics", The 47th National Congress of Information Processing Society of Japan 